\title{
Interdisciplinarity in action: Using infrared thermography to teach plants' energy balance in secondary education
}

\author{
by J. Marques da Silva*, P. Correia*, M. Calejo Pires*, J. Soares Augusto**, J. Miguel Costa**
}

\author{
*BiolSI - Biosystems and Integrative Sciences Institute, Faculdade de Ciências, Universidade de Lisboa. \\ Campo Grande, 1749-016 Lisboa, Portugal, imlsilva@fc.ul.pt \\ **Inesc-ID, Lisboa and Departamento de Física, Faculdade de Ciências, Universidade de Lisboa. Campo \\ Grande, 1749-016 Lisboa, Portugal. \\ ***Departamento de Ciências e Engenharia de Biossistemas, Instituto Superior de Agronomia, Universidade de \\ Lisboa, Tapada da Ajuda, 1349-017 Lisboa, Portugal, miguelcosta@isa.ulisboa.pt
}

\begin{abstract}
Thermography emerged as a practical tool to teach plant biology in higher education. Indeed, thermography can help to teach basic principles of plants' physiology such as their energy balance. The well-established fact that increased leaf temperature reflects stomatal closure, being therefore a measure of plants' response to drought, supports the relevance of the technique. Nowadays, affordable thermographic cameras allow to extend the use of this technology to secondary education. To achieve this goal, however, training high school science teachers is instrumental. We herein report and discuss a training action on the principles of thermography and their application to plant biology.
\end{abstract}

\section{Introduction}

\subsection{Infrared radiaton and the electromagnetic spectrum}

Electromagnetic radiation is a form of electromagnetic energy that propagates in space as waves (synchronized oscillations of electric and magnetic fields). The spectrum of the electromagnetic radiation comprises variable wavelength bands and the correspondent frequency ranges, which in turn imparts different characteristics of radiation. Examples of bands within the electromagnetic spectrum include $x$-rays (most widely clinically used to treat malignant lesions), visible light, infrared (IR) light, and radio waves mostly used for communications (Fig. 1).

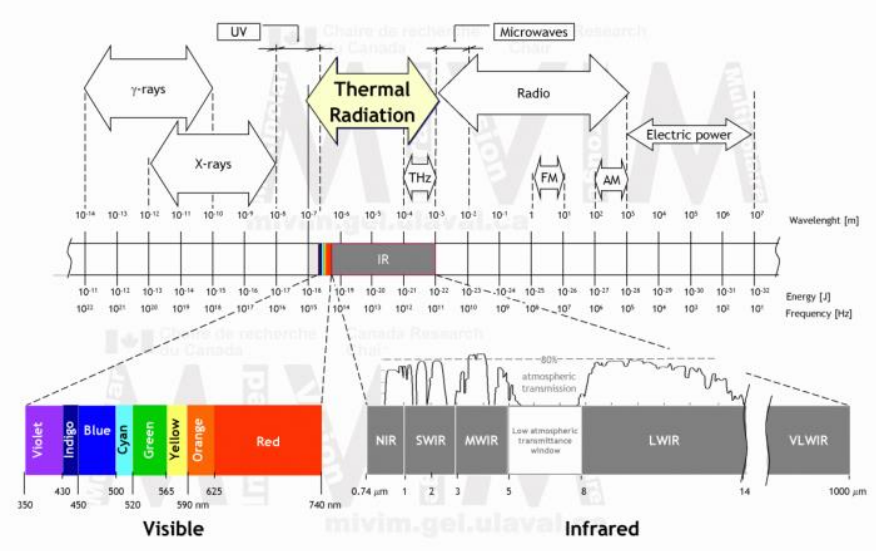

Fig. 1: The electromagnetic spectrum, highlighting the visible and the infrared radiation bands (image licensed under the Creative Commons Attribution-Share Alike 3.0 Unported license. Attribution: Ibarrac at English Wikipedia)

When using thermography, we are dealing with a specific wavelength region of the electromagnetic spectrum, the infrared thermal band.

\subsection{The use of thermography for educational purposes}

The use of thermography to support educational purposes is not new [1, 2]. Indeed, IR Thermography (infrared thermal imaging) emerged as a cheap and a user-friendly tool to support the industry, science, and education. 
Thermography can suit as a tool to teach optics and radiation physics but also to teach students on how plants and animals "work" - i.e., plant and animal physiology. Plant science and the agroindustry make use of imaging systems such as thermography to study and monitor plants/crops at different levels (from an individual leaf to an entire plant, field, or region) [3-5]. The use of thermography in plant science [6] emerged as a feasible approach to characterize plant-environment interactions (water relations, drought stress) in open field [7] or controlled environments (lab, phytotron, greenhouse). The use of thermography in plant science and agronomy relates to the well-established principle that increased leaf temperature

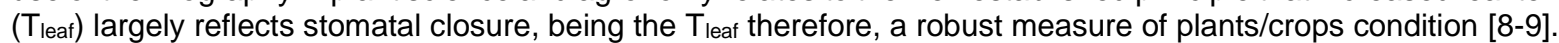

Study of biophysics and thermal dynamics in plants has been largely based on point measures of temperature with IR thermometers and thermocouples (detecting heat conduction) or pyrometers (detecting heat radiation). Thermal imaging by means of an IR camera provides a real-time, holistic image $[9,10]$. Besides information on plant/crop performance, IR imaging can help to "visualize", in real-time, temperature variation across bodies/surfaces, which may have physiological meaning. As a result, thermography can support education, helping students to envisage and better comprehend biophysical or ecophysiological phenomena involving heat exchange.

\subsection{The physics behind thermography and IR radiation emission}

The basics of the thermography technique is that all bodies above $0^{\circ} \mathrm{K}\left(-273^{\circ} \mathrm{C}\right)$ emit electromagnetic radiation, and the warmer they are the more radiation they will emit according to the Stefan-Boltzmann law:

$$
\mathrm{J}=\varepsilon \sigma\left(\mathrm{T}_{\text {leaf }}\right)^{4},
$$

where, $\mathrm{J}$ is the total radiated power per unit of area emitted by the object, $\varepsilon$ is the emissivity of the material (e.g. leaf), $\sigma$ is the Boltzman constant and $T_{\text {leaf }}$ is the temperature of the leaf.

In IR thermography, only a part of the infrared band of the electromagnetic spectrum (usually wavelengths in the range of 7 to $14 \mu \mathrm{m}$ ) is used to measure the temperature. The thermal imaging cameras, equipped with the appropriate IR sensors, create an image of the target surface, based, not on the amount of visible light emitted, but on the amount of IR radiation emitted from each point of the surface, depending on that point temperature $(T)$; because IR radiation is invisible to the naked eye, a built-in software converts the different energy intensity levels into different colours, so producing a (visible) false colour image.

However, the amount of radiant power emitted and/or collected does not depend only on the temperature $\left(\mathrm{T}^{4}\right)$; it depends also:

- $\quad$ on the optical properties of the surface material, mainly the material emissivity $\varepsilon$, that might have different values between 0 and 1 ; for example, low values for polished metals $(\varepsilon=0.2$ for silver) or high values for ice $(\varepsilon=0.98)$ or organic materials such as plants' leaves $(\varepsilon \sim 0.99)$

- on the conditions of the surrounding ambient, such as air temperature and humidity

- on the distance from the imaged surface to the camera

In consequence, to have accurate measurements of temperature based on the IR radiation collected from a surface, a calibration is required for emissivity, distance, and temperature and humidity of the surrounding media. Nevertheless, for points in a surface that are approximately at equal distance from the camera, and with the same levels of ambient temperature and relative humidity, the radiation detected from different areas of the surface is only dependent on the specific emissivity and/or the temperature. Since the amount of radiation emitted by a surface increases with temperature, IR thermography allows to see variations in temperature of the surface.

\subsection{How plants control their temperature}

Plants are sessile organisms and therefore cannot avoid exposure to stressful sub-optimal or deleterious temperatures [11]. Therefore, they are extremely dependent on physiological mechanisms of body temperature control. Plant's leaves are particularly exposed to environmental temperature and, since they are highly metabolic active organs, leaves' temperature control is critical. At a certain moment in time, the leaf energy balance is the difference between all the environmental energy inputs (into a leaf) and all energy output (from the leaf) (Fig. 2). 


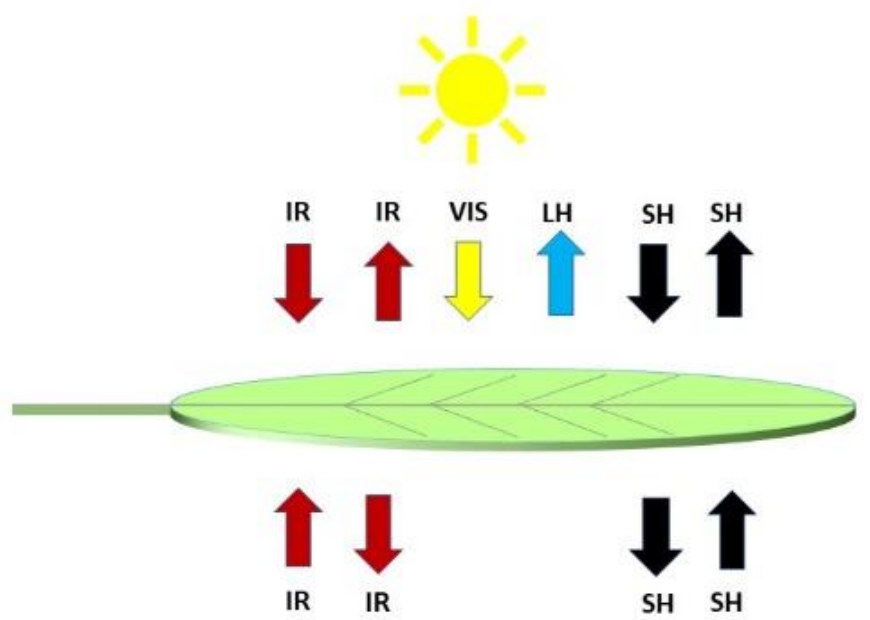

Fig. 2: Leaf energy balance (simplified); IR, infrared radiation; VIS, visible radiation; $\mathrm{SH}$, sensible heat (convection and conduction); LH, latent heat.

The difference is energy accumulated or spent on metabolic activities and, mostly, changes in leaf temperature [12]. The energy input cames from solar radiation (IR and visible), and IR radiation absorbed from the surroundings. The energy output is emitted IR radiation, heat convection and heat conduction (sensible heat), and heat loss accompanying water evaporation (latent heat). This latter component may be controlled by plants, as they are able to control transpiration by regulating stomatal aperture. Stomata are tiny pores in leaf (and other aerial organs) epidermis, through which most gas exchanges between plant and the surrounding atmosphere occur. The stomatal pore has a pair of specialized cells the guard cells - which control stomatal aperture. When the guard cells are fully turgid, stomata will be fully open, and they close when guard cells are plasmolyzed. Intermediate guard cell turgor will lead intermediate stomatal aperture. Guard cell turgor is determined by the cell concentration of osmolytes, which is regulated by a complex metabolic network [13]. Stomatal control is critical to attain the necessary trade-off between leaf temperature regulation, water saving, and carbon dioxide input for photosynthesis. Since thermography can easily determine the temperature of leaf's surface it is already used to teach the principles of plants' energy balance to higher education students, both in biology (e.g., at the Faculty of Sciences, University of Lisbon) and agronomy courses. The well-established fact that increased leaf temperature reflects mostly stomatal closure, being therefore a robust measure of plants and crops response to drought, supports the relevance of this technique.

\subsection{Different sensors, different resolution, and different cost: new opportunities}

The pedagogical potential of thermography for biology education is well described in literature [1, 14] and, nowadays, we assist to the emergence of cheaper and increasingly easy to operate thermographic cameras (e.g. in mobile phones) available for a wide range of activities, namely teaching of plants function. However, to achieve this goal, and fully take advantage of this imaging tool, training high school science teachers is instrumental and highly needed to improve efficiency in the use of cameras and related experimental demonstrations. In this article we report and discuss a training activity carried out for high school science teachers focused on the principles of thermography and their application to plant biology. The action was designed for half a day and 20 trainees and was jointly implemented by a physicist and a 
biologist/agronomist. Four main goals were established: a) To teach basic physical principles of thermography, to present the IR imaging technology and to alert to the basic operational aspects in the use of a thermographic cameras; $b$ ) To present examples of the use of thermography in both agronomic and plant sciences; c) To show the potential of thermography for educational purposes in different areas of teaching (e.g. physics, plant physiology and agronomy, etc.); and d) To alert to the limitations of the technique and equipment when monitoring certain aspects of plant ecophysiology (detection of water stress, selection of genotypes with relevant characteristics, etc.). Therefore, tha action started with a theoretical introduction, comprising a) the physics of thermography, b) the theory of leaf energy balance (e.g. the role of stomatal regulation) and c) the potential of thermography to teach biology to secondary students. Afterwards, the trainees performed several hands-on experiment, following a detailed protocol.

\section{Materials and methods}

After the theoretical introduction,the trainees formed 4 groups, and were able to test different cameras, ranging from simple and low cost models (Flir One (7-14 $\mu \mathrm{m}, 60 \times 80$ pixels)) to more sophisticated and expensive models (Flir

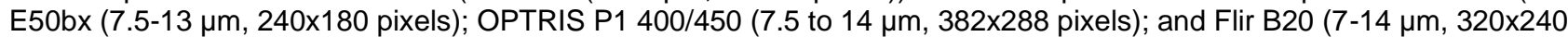
pixels)). A detailed experimental protocol was implemented and included two major sets of experiments.

1) Experimental set 1: used inorganic materials and aimed to highlight the physics underlying thermography.

2) Experimental set 2: used plants of Arabidopsis thaliana (wild-type (WT) and stomatal mutants) to illustrate the role of stomatal control on transpiration and leaf energy balance.

In the Experimental set 1, it was used:

a bath of water with ice for the adjustment of the zero of the equipment, being $0^{\circ}=273 \mathrm{~K}$ the temperature of the triple point of water;

pieces of insulation tape on a card board to distinguish the visible radiation emitted, detected with naked eye, and the IR radiation emitted, not visible, but detected by the IR cameras

Objects with different emissivity values, in this case, plant leaves and pieces of metal

Samples of wet and dry soil, to illustrate the cooling effect of evaporation

In the Experimental set 2, it was used:

Arabidopsis plants, watered and drought stressed, wild-type and stomatal mutants

\section{Results}

A total of five experiments were done in Set 1. In Exp. 1, a simple temperature measurement of melting ice was done (Fig. $3 \mathrm{~A}$ ) to introduce thermography to the trainees, by measuring a body with a known temperature (melting ice, 0 ${ }^{\circ} \mathrm{C}$ ). In Exp. 2 two contrasting materials, aluminum (low infrared emissivity, $\varepsilon=0,05$ ) and plant tissue (high infrared emissivity, $\varepsilon=0.95-0.97$ ) were compared at ambient temperature (Fig 3B). Thermal images show differences due to different material's infrared emissivity $(\varepsilon)$. Since in the thermal camera infrared emissivity was set to $0.95-0.96$, we got an accurate measurement of the $T_{\text {leaf, }}$ whereas the temperature of aluminum foil was highly underestimate, showing the limitations of thermography to measure temperature of highly reflective materials such as metals. In Exp. 3, the effect of object properties (color) was explored. Plastic tapes with different colors (Fig. 3C) were observed under dim and strong light. As expected, under dim light the tapes presented identical temperature, showing that color had no effect on temperature (result not shown). However, under more intense light, darker tapes exhibit slightly higher temperatures, due to a higher absorptivity of radiation (Fig. 3D). In Exp. 4 heat transfer phenomena were illustrated. In the first procedure, trainees collected a thermographic image of the bench before (Fig. 3E) and after (Fig. 3F) pressing their hands on it to illustrate heat transfer (conduction) from the human body to the bench material. The heat signature of three fingerprints can be clearly seen in Fig. 3F, in contrast with Fig. 3E, where they are absent. The mean temperature of the bench was $28.4^{\circ} \mathrm{C}(\mathrm{Fig} .3 \mathrm{~F})$ but the contact with a warmer object (participant's palm of the hand, circa $36.5^{\circ} \mathrm{C}$ ) conducted heat to the bench, locally increasing its temperature to $31.2^{\circ} \mathrm{C}$.

In Exp. 5 evaporative cooling was demonstrated by comparing the temperatures of a dry and a wet paper sheet. The sheet wetted with distilled water had lower temperature (less $3.7^{\circ} \mathrm{C}$ ) than the one that was kept dry (Fig. $3 G$ ) Evaporative cooling was also demonstrated in an experiment using dry and wet soil, making the transition between the first set of experiments, with inert materials, to the second set of experiments, with Arabidopsis plants. Figure $3 \mathrm{H}$ shows that the soil in watered pot presents lower temperature than the soil in a non-irrigated pot. 


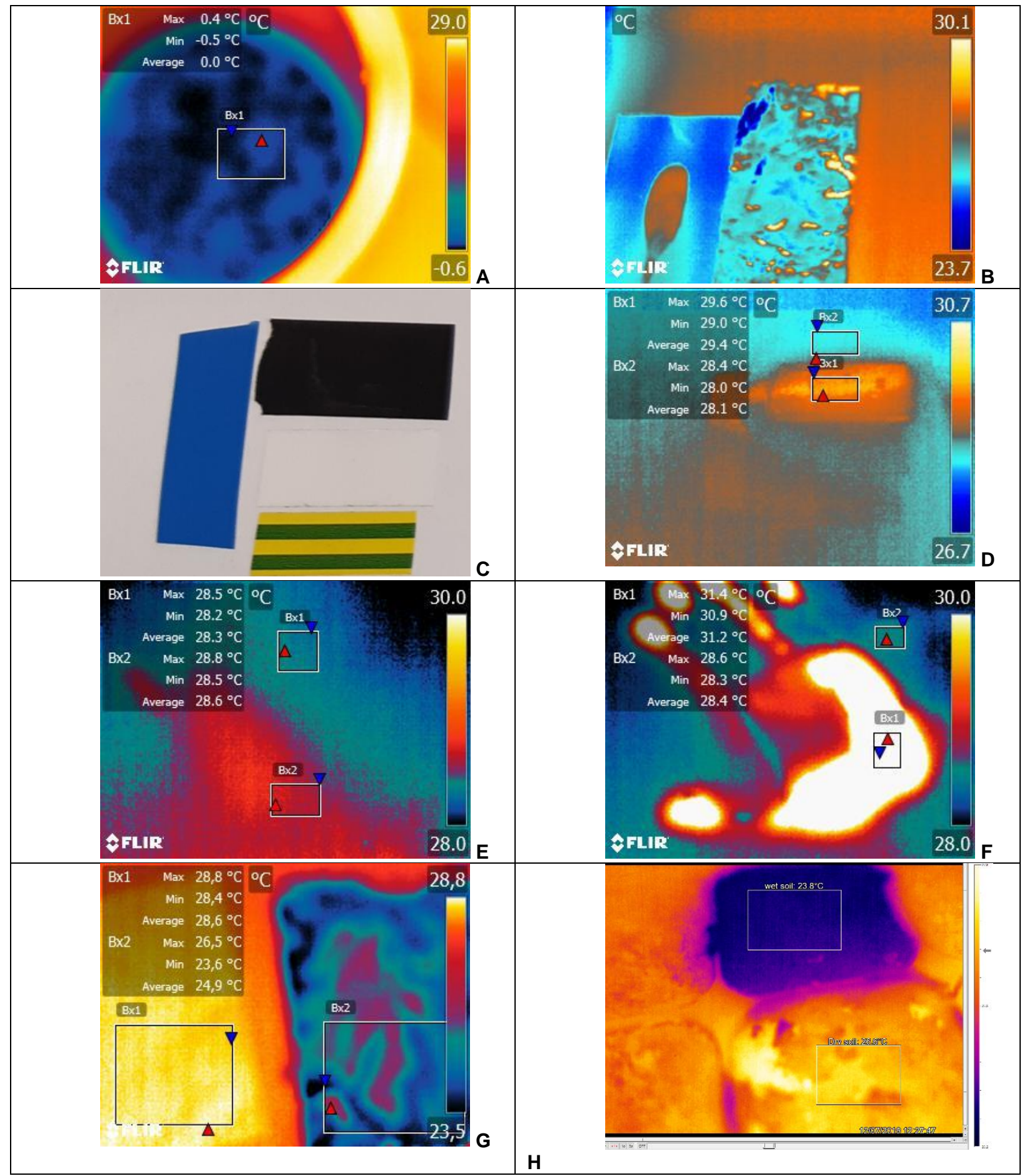

Fig. 3. $R G B(C)$ and false colored thermal images (Flir E50bx, 7.5-13 $\mu \mathrm{m}, 240 \times 180$ pixels: $B$; ) of A) melting ice in a goblet; $B$ ) an aluminum foil (right) and a detached leaf of Arabidopsis thaliana) (left); C) RGB image of a set of different color plastic tape pieces and the corresponding D) thermal image; E) bench surface and F) bench surface after hand's contact; G) dry (left) and wetted (right) paper stripes; and H) pots with dry (down) and wet (up) soil. 
Stomatal mutants exhibit changes on the signalling pathways controlling stomatal function and regulation (Fig. 4A). The WT plants (left) had a cooler T leat than the mutants (right) characterized by more closed stomata (Figs. 4B). This illustrates the role of stomata on leaf energy balance and leaf thermal regulation. The role of stomata on transpiration and leaf evaporative cooling is also nicely illustrated in an experiment where leaf surface is covered with a thin layer of petroleum gel (grease) (Fig. 4C). We can see that the Arabidopsis leaf covered with petroleum gel (left) has a higher temperature $\left(+1.4^{\circ} \mathrm{C}\right)$ that the untreated control leaf. The layer of impermeable petroleum gel obstructs the stomatal pores, hindering transpiration and therefore evaporative cooling. In conclusion using thermography to study biological phenomena combines physics and biology and also points out the large potential of imaging tools and interdisciplinary interaction, to explain and describe plant behavior and functioning to students in a more comprehensive way.

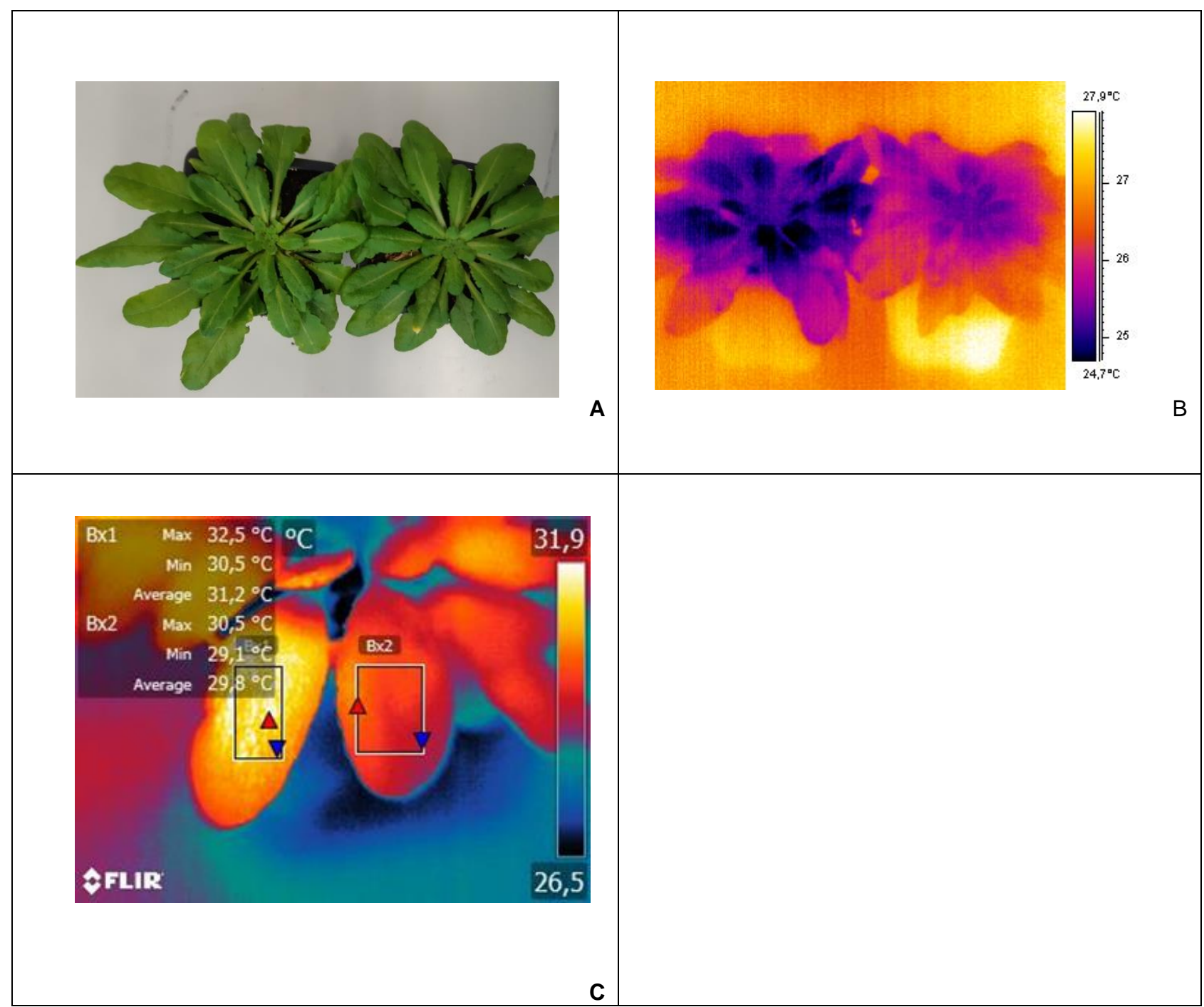

Fig. 4. RGB (A) and false colored thermal images (B: Flir B20, 7-14 $\mu \mathrm{m}$, 320x240 pixels; C: Flir E50bx, 7.5-13 $\mu \mathrm{m}, 240 \times 180$ pixels) of $A$ ) wild-type (left) and stomatal mutant (right) of Arabidopsis thaliana and $B$ ) corresponding thermal image from the wild-type (cooler, bluish colors) (right) and the stomatal mutant, with more closed stomata (warmer, brighter colors) (left); and C) greased (left) and non-greased (right) leaves of Arabidopsis thaliana. 


\section{Aknowledgments}

A part of this research was funded by Fundação para a Ciência e Tecnologia, FCT/MCTES/PIDDAC, Portugal, through the projects UIDB/04046/2020 and UIDP/04046/2020 (BiolSI). This work is supported by the Fundação para a Ciência e Tecnologia through the Research Project INTERPHENO - "Fostering High Throughput Plant Phenotyping by an Interdisciplinary Approach" (PTDC/ASP-PLA/28726/2017) and the BiolSI internal projects Optigrape I and II.

\section{REFERENCES}

[1] Xie C. "Transforming science education with IR imaging". Paper presented at the InfraMation 2012, Orlando, FL, November 6-8, 2012

[2] Vollmer M., Möllmann K.-P. "Teaching physics and understanding infrared thermal imaging". 14th Conference on Education and Training in Optics and Photonics: ETOP 2017, edited by Xu Liu, Xi-Cheng Zhang, Proc. of SPIE Vol. 10452, 104522C, 2017. doi: 10.1117/12.2266142

[3] Patel N.R., Anapashsha R., Kumar S., Saha S.K., Dadhwal V.K. "Assessing potential of MODIS derived temperature/vegetation condition index (TVDI) to infer soil moisture status", International Journal of Remote Sensing, 30:1, 23-39, 2009. DOI: 10.1080/01431160802108497

[4] Costa J.M., Marques da Silva J., Pinheiro C., Barón M., Mylona P., Centritto M., Haworth M., Loreto F., Uzilday B., Turkan I., Oliveira M.M. "Opportunities and Limitations of Crop Phenotyping in Southern European Countries". Frontiers in Plant Science, 10 (September), 1-16, 2019.

[5] Sagan V., Maimaitijiang M., Sidike P., Eblimit K., Peterson K.T., Hartling S., Esposito F., Khanal K., Newcomb M., Pauli D., Ward R., Fritschi F., Shakoor N., Mockler T. "UAV-Based High Resolution Thermal Imaging for Vegetation Monitoring, and Plant Phenotyping Using ICI 8640 P, FLIR Vue Pro R 640, and thermoMap Cameras". Remote Sens. 11, 330, 2019.

[6] Hashimoto Y., Ino T., Kramer P.J., Naylor A.W., Strain B.R. "Dynamic Analysis of Water Stress of Sunflower Leaves by Means of a Thermal Image Processing System". Plant Physiol. 76, 266-269, 1984.

[7] Zovko M., Boras I., Švaić S. "Assessing plant water status from infrared thermography for irrigation management". QIRT 2018 Proceedings, 507-501, 2018.

[8] Jones H.G. "Thermal imaging and infrared sensing in plant ecophysiology". Advances in Plant Ecophysiology Techniques, 135-151, 2004.

[9] Costa J.M., Grant O.M., Chaves M.M. "Thermography to explore plant- environment interactions". Journal of Experimental Botany, 64(13), 3937-3949, 2013.

[10] Melander E., Haglund J., Weiszflog M., Andersson S. "More than Meets the Eye - Infrared Cameras in OpenEnded University Thermodynamics Labs". The Physics Teacher 54, 528, 2016. https://doi.org/10.1119/1.4967889

[11] Duque A.S., Almeida A.M., Bernardes da Silva A., Marques da Silva J., Farinha A.P., Santos D., Fevereiro P., Araújo S.S. "Abiotic Stress Responses in Plants: Unravelling the Complexity of Genes and Networks to Survive". In: Abiotic Stress - Plant Responses and Applications in Agriculture (Kourosh Vahdati and Charles Leslie eds.), chap. 3, pp. 49-101, InTech, Rijeka, Croatia, 2013.

[12] Nobel P.S. "Physicochemical and Environmental Plant Physiology" (3rd ed.), Elsevier / Academic Press, San Diego, 2005.

[13] Lawson T., Matthews J. "Guard Cell Metabolism and Stomatal Function". Annu. Rev. Plant Biol. 7, :273-302, 2020. https://doi.org/10.1146/annurev-arplant-050718100251

[14] Haglund J., Schönborn J.J. "The Pedagogical Potential of Infrared Cameras in Biology Education”. The American Biology Teacher, 81 (7), 520-523, 2019. 